\title{
Invited review: A systematic review and qualitative analysis of treatments other than conventional antimicrobials for clinical mastitis in dairy cows
}

\author{
D. Francoz, ${ }^{*}$ V. Wellemans, ${ }^{*}$ J. P. Dupré, ${ }^{*}$ J. P. Roy, ${ }^{\star} \dagger$ F. Labelle, $\ddagger$ P. Lacasse, $§ \S$ and S. Dufour†\# ${ }^{1}$ \\ *Département de Sciences Cliniques, Faculté de Médecine Vétérinaire, Université de Montréal, C.P. 5000, St-Hyacinthe, QC, J2S 7C6, Canada \\ †Canadian Bovine Mastitis and Milk Quality Research Network, C.P. 5000, St-Hyacinthe, QC, J2S 7C6, Canada \\ ¥Valacta, 555 Boul. des Anciens-Combattants, Ste-Anne-de-Bellevue, QC, H9X 3R4, Canada \\ $\S$ Sherbrooke Research and Development Centre, Agriculture and Agri-Food Canada, 2000. Collège, Sherbrooke, QC, J1M 0C8, Canada \\ \#Département de Pathologie et Microbiologie, Faculté de Médecine Vétérinaire, Université de Montréal, C.P. 5000, St-Hyacinthe, QC, J2S 7C6, \\ Canada
}

\section{ABSTRACT}

Clinical mastitis is an important disease in dairies. Its treatment is mainly based on the use of antimicrobial drugs. Numerous non-antimicrobial drugs and treatment strategies have already been reported for clinical mastitis treatment, but data on their efficacy have never been collated in a systematic way. The objective of this systematic review was to identify treatments other than conventional antimicrobials for the treatment of clinical mastitis in lactating dairy cows. A systematic review was performed with studies written in English or French selected from CAB Abstracts, PubMed, and Web of Science from January 1970 to June 2014. Controlled clinical trials, observational studies, and experimental challenges were retained. Lactating dairy cows with clinical mastitis were the participant of interest. All treatments other than conventional antimicrobials for clinical mastitis during lactation were retained. Only studies comparing the treatment under investigation to a negative or positive control, or both, were included. Outcomes evaluated were clinical and bacteriological cure rates and milk production. Selection of the study, data extraction, and assessment of risk of bias was performed by 3 reviewers. Assessment of risk of bias was evaluated using the Cochrane Collaboration tool for systematic review of interventions. A total of 2,451 manuscripts were first identified and 39 manuscripts corresponding to 41 studies were included. Among these, 22 were clinical trials, 18 were experimental studies, and 1 was an observational study. The treatments evaluated were conventional anti-inflammatory drugs $(\mathrm{n}=14)$, oxytocin with or without frequent milk out

\footnotetext{
Received December 23, 2016.

Accepted May 24, 2017.

${ }^{1}$ Corresponding author: simon.dufour@umontreal.ca
}

$(\mathrm{n}=5)$, biologics $(\mathrm{n}=9)$, homeopathy $(\mathrm{n}=5)$, botanicals $(n=4)$, probiotics $(n=2)$, and other alternative products $(n=2)$. All trials had at least one unclear or high risk of bias. Most trials $(n=13)$ did not observe significant differences in clinical or bacteriological cure rates in comparison with negative or positive controls. Few studies evaluated the effect of treatment on milk yield. In general, the power of the different studies was very low, thus precluding conclusions on noninferiority or nonsuperiority of the treatments investigated. No evidence-based recommendations could be given for the use of an alternative or non-antimicrobial conventional treatment for clinical mastitis. However, probiotics and oxytocin with or without frequent milk out should not be recommended. We concluded that homeopathic treatments are not efficient for management of clinical mastitis.

Key words: systematic review, lactating dairy cow, clinical mastitis, non-antimicrobial therapy

\section{INTRODUCTION}

Clinical mastitis (CM) has important deleterious effects on dairy herd productivity, longevity, and profitability because of decreased milk production, decreased reproductive performance, costs associated with treatments, and the increased risk of culling and death of affected animals (Halasa et al., 2007). In 2 recent studies conducted on Canadian dairies, most the participants (71 and 54\%) stated that they were worried by the cost of mastitis on their farm (Dufour et al., 2010; Francoz et al., 2011). Mastitis was reported to be the most important cause of antimicrobial drug use in dairy herds with close to half of the antimicrobials used on dairies being used for mastitis control, mainly for treatment of CM cases (Saini et al., 2012).

For certification, organic dairies have to meet specific requirements and the use of conventional treatments 
(i.e., antimicrobials) is limited. In cases of clinical diseases, alternative treatments must be instituted first and conventional treatments must only be used when alternative treatments are not successful (Agriculture and Agri-Food Canada, 2012). Moreover, the use of antimicrobials are also scrutinized in conventional dairies and even restricted in some European countries (Santman-Berends et al., 2016). Consequently, conventional dairy producers also have a growing interest in non-antimicrobial therapies.

The use of numerous non-antimicrobials CM treatments has been reported in organic and in conventional dairies. For simplicity, in the remainder of the manuscript non-antimicrobial treatments will be divided in 2 categories: (1) alternative treatments (i.e., alternative to the mainstream allopathic medicine); and (2) non-antimicrobial conventional treatments (i.e., mainstream allopathic treatments that do not directly affect microorganisms). Alternative treatments mainly include homeopathy, biologics (products derived from live organisms that can be used to treat or prevent a disease), botanicals (i.e., aromatherapy, phytotherapy), probiotics, and acupuncture (Karreman, 2009; Ruegg, 2009; Ameloot, 2010).

Evaluation of CM treatment efficacy is a difficult task. First, efficacy can be evaluated based on clinical or bacteriological cures, or both. Furthermore, the effect on milk production is important for determining marketability of a treatment. Second, following initial in vitro and in vivo experimental studies, treatment efficacy should ultimately be evaluated in a randomized controlled trial (RCT; i.e., the gold standard for evaluating the effect of medical treatments). For CM treatment, because a relatively high rate of spontaneous resolution is expected (>30\%; (Hektoen et al., 2004)), the treatment under investigation must be compared with one or many control groups. Optimally, at least 3 different treatment groups would be included in a RCT: a negative control group (no treatment; when justifiable, for instance for less severe cases), a positive control group receiving currently recommended treatment (an antimicrobial, for instance), and the tested treatment group (the non-antimicrobial treatment in this case). Moreover, persons responsible for treatment administration and for evaluation of health outcomes, caregivers, and analysts should be blinded to group allocation. To date, very few alternative treatments have been formally evaluated in RCT. Consequently, recommendations frequently conveyed in the dairy organic sector on use of some alternative treatments are not based on data of efficacy and are often questionable. Additionally, results on the efficacy of these approaches for CM treatment have never been collated in a coherent whole and compared. To our knowledge, only 2 narrative literature reviews have been published on CM alternative treatment (Ruegg, 2009; Ameloot, 2010). These reviews were not systematic reviews (SR) or meta-analyses (Sargeant et al., 2006), and all the different alternative products were not reviewed. Thus, the efficacy of the non-antimicrobial CM treatments available have never been evaluated in a systematic manner. Providing evidence-based advice on non-antimicrobial treatments of CM is, therefore, a difficult task for dairy practitioners and extension agents. A rigorous and exhaustive evaluation of these treatments would help inform the development of standard operating procedures for the treatment of CM in organic dairy. Furthermore, alternative treatments showing unequivocal efficacy could also be used in conventional dairies and would potentially help decrease use of antimicrobials.

Therefore, the objectives of the current study were to identify and report efficacy results of alternative and non-antimicrobial conventional therapies for treatment of CM in dairy cows that have already been studied in RCT, non-RCT, observational studies, or experimental studies using mastitis models. The aim of the study was to determine the therapies that have demonstrated efficacy in scientifically sound research protocols or that have shown consistent results across the available literature, or both.

\section{MATERIALS AND METHODS}

The chosen study design was a SR of the literature. Systematic reviews aim at collecting, analyzing, and synthesizing multiple research studies. This methodology can provide an exhaustive summary of the current literature on a given topic using a detailed and reproducible methodology.

\section{Criteria for Considering Studies for the Review}

Types of Studies. Study designs such as RCT, nonRCT, observational studies (cohort, cross-sectional, and case-control designs), and experimental challenges using naturally acquired CM or bacterial- or endotoxininduced CM models were retained. For studies using bacterial- or endotoxin-induced CM models (i.e., experimental challenge), to be included in the review the treatment had to be instituted after onset of clinical signs (vs. the treatment being administered at the time of the challenge or shortly after). Study designs such as case-series, case-report, or expert opinion were not retained. Only studies evaluating the efficacy of a treatment other than conventional antimicrobials for treatment of CM (grade 1 to 3 ) during the lactating period were included. All studies on the treatment of subclinical mastitis or on dry cow therapy were excluded. 
Types of Participants. Lactating dairy cows with $\mathrm{CM}$ were the participants of interest. Because extrapolation from other species is difficult and because of the difference in pathogens involved or immune system function, tropical breed (e.g., Gyr cow), other ruminant (e.g., water buffalo), and nonruminant species were excluded.

Types of Interventions. All treatments other than conventional antimicrobials for treating $\mathrm{CM}$ in lactating dairy cows were considered. These treatments could have been administered locally on the mammary gland by topical or intrammammary administration, or systemically, by oral, intra-vulvar, intra-nasal, i.m., s.c., or i.v. administration. Trials or control groups where a combined intervention consisting of a non-antimicrobial and an antimicrobial treatment was investigated were excluded. Control groups could include a group of untreated cows or a group of cows receiving placebo (i.e., negative controls) or a group of cows receiving a conventional antimicrobial intervention (i.e., positive control). Studies making use of multiple control groups were also retained. Studies not using at least one control group were considered to be case-series or case-report studies and were excluded.

Types of Outcome Measured. When evaluating efficacy of a CM treatment, one would expect results on resolution of clinical signs (i.e., clinical cure), resolution of the IMI (i.e., bacteriological cure), and, possibly, on the effect of the treatment on subsequent milk production. Consequently, the primary outcomes evaluated in this study were (1) resolution and recurrence of clinical signs, which include systemic (rectal temperature, heart rate, respiratory rate, rumen motility, ruminal contraction rate, attitude, appetite, or behavior score) as well as local signs (quarter size, quarter edema, quarter inflammation, milk appearance) and milk SCC variation; (2) bacteriological cure; and (3) milk production (short and long term).

\section{Search Methods for Identification of Studies}

Electronic Search. Identification of the relevant databases and elaboration of the search strategy were conducted with the help of a librarian (Huguette Mallette, Faculté de médecine vétérinaire, Université de Montréal). The initial database search was conducted to identify relevant literature irrespective of language and publication status. Two databases, CAB Abstracts (http://ovidsp.ovid.com/autologin.cgi), and PubMed (https://www.ncbi.nlm.nih.gov/pubmed), and 1 search platform, Web of Science (https://login.webofknowledge.com), were searched on the same day (June 3, 2014) for original research published on or after January 1, 1970. Boolean search strategies using thesaurus terms or natural language (or both) used in abstract, title, original title, broad terms, or heading and based on descriptors of the population of interest (dairy cows), outcome studied (CM), and the treatment aspect (the treatment other than conventional antimicrobials), were designed specifically for each database. Regarding the treatment aspect, an exhaustive list of search terms was developed by the authors who were experts on this topic. Key words and search strategies used for each database are presented in the Appendix.

Searching Other Resources. When a publication was retained to be included in the SR after having undergone the complete selection process (see below), the list of references of the included publication was then reviewed by 2 reviewers (DF and VW) to identify additional publications not previously identified by the initial database search. These additional publications were also evaluated for inclusion by 2 reviewers (DF and VW) as described for the initial electronic search, but without formal blinding (see below). Whenever this process resulted in the inclusion of an additional publication, the list of references of the newly included publication was again screened and the process was repeated until complete depletion.

\section{Data Collection and Analysis}

Selection of Studies. Results of the 3 electronic database searches were imported in EndNote X7.1 (EndNote, Philadelphia, PA), and duplicates were eliminated. Three reviewers (JPD, VW, and DF) worked independently to evaluate each title and abstract. During this process, reviewers were blinded to authors' names, journal, and year of publication of the manuscripts. At this stage, reviewers were more liberal for inclusion of publications (i.e., in case of doubt, publication was retained for further evaluation). The results of the review process of the 3 reviewers were compared, and whenever the reviewers disagreed on the selection of an abstract, a consensual decision was taken. Full text of the articles selected were then obtained and reviewed by 4 reviewers (JPD, VW, DF, and SD) in a similar fashion, but with strict application of the inclusions and exclusions criteria. To be included, manuscript had to (1) be published between 1970 and June 2014; (2) be written in English or in French; (3) evaluate a treatment other than a conventional antimicrobial treatment for $\mathrm{CM}$ on dairy lactating cows (i.e., prevention, dry cow therapy, or treatment of subclinical mastitis were excluded as well as studies conducted on tropical breeds and buffaloes); (4) present original research (i.e., case series, case report, and reviews by experts were excluded); (5) be conducted in vivo on dairy cows; (6) have a treatment instigated after, not before, apparition of clinical signs; 
and (7) present results on clinical cure (e.g., resolution of clinical signs, CM recurrence), bacteriological cure (e.g., posttreatment milk bacteriological culture), the return of milk SCC at an acceptable level, or milk yield following treatment.

If 2 or more publications presented results from the same study, only the most complete study was selected. In some instances, authors were contacted to confirm originality of results from different publications.

Data Extraction and Management. Data of each selected publication were extracted independently by 3 reviewers (SD, VW, DF) using a standardized form. The following information was extracted: (1) Study methods and characteristics (year of publication, country of study, funding, design of the study (including randomization and blindness); (2) participants (number of herds, number of cows, number of CM cases, age, breed, and inclusion and exclusion criteria including diagnostic criteria for $\mathrm{CM}$ ); (3) intervention (type of non-antimicrobial treatment, dose, route of administration, frequency of administration, and duration of treatment); (4) control group (no treatment, placebo, positive antimicrobial control treatment); (5) outcomes (types of outcome measures, reported outcomes, adverse events, follow-up time, and results). All extracted data were compared between the 3 reviewers for completeness and accuracy. Data were then transferred into Review Manager 5.3 (The Nordic Cochrane Centre, The Cochrane Collaboration, Copenhagen, Denmark).

Assessment of Risk of Bias in Included Studies. Assessment of risk of bias in included studies was evaluated using The Cochrane Collaboration's tool for assessing risk of bias (Cochrane Handbook for Systematic Reviews of Interventions version 5.1.0). This tool was available through Review Manager (RevMan 5.3). It included the appraisal of 7 specific sources of bias commonly observed in clinical trials: random sequence generation and allocation concealment (2 potential sources of selection bias); blinding of participants and personnel (a potential source of performance bias); blinding of outcome assessment (a potential source of detection bias); incomplete outcome data (a potential source of attrition bias); selective outcome reporting (a potential source of reporting bias); and any other potential bias not explicitly listed. Risk of biases was first assessed independently by 3 reviewers (SD, VW, DF) as low risk, high risk, or unclear risk. It is important to note that the unclear risk category was not used as an intermediate category between the low and high risk categories. This category was instead used whenever the information presented was insufficient to permit risk evaluation. Results of the 3 evaluations were then compared and a consensus was obtained in cases of disagreement.
Statistical Analyses. Whenever clinical cure or bacteriological cure rates were compared in selected publications, a post hoc statistical power analysis was conducted. For these analyses, the power of the study to conclude on statistical significance regarding the observed difference between treatment and positive control groups or between treatment and negative control groups, given the available group's size, and using an $\alpha$ of 0.05 , was computed using the Fisher exact test for 2 proportions available in SAS 9.4 Power procedure (SAS Institute Inc., Cary, NC). These post hoc power calculations were used to better appraise nonsignificant results. For instance, a study with a relatively small sample size and for which large differences in cure rate were observed between the treatment under investigation and a group of cows treated with a conventional CM treatment could report no statistical difference in proportion of cured animals and, thus, wrongly conclude that the investigated treatment is equivalent to the conventional treatment. Similarly, a relatively small sample size could wrongly lead to the conclusion of no difference between the treatment under investigation and a placebo, if the clinical effect of the treatment is relatively modest. Reporting the power of these studies would then help appraise and interpret the clinical significance of nonsignificant results.

\section{RESULTS}

\section{Description of Studies}

Search Results. Result of the different steps for identifying the relevant literature is presented in Figure 1. Briefly, 2,451 independent abstracts were identified using the search strategy previously presented. Among the 167 papers remaining after title or abstract evaluation (or both), 54 were excluded before complete text evaluation: 48 were written in a language other than English or French (Russian 13, German 11, Chinese 8, Polish 5, Portuguese 3, Spanish 2, Arabic 1, Czech 1, Hungarian 1, Italian 1, Norwegian 1, and Swedish 1) and full text was not available for 6 publications (i.e., abstract only). Among the 113 manuscripts reviewed, 78 were excluded after full text assessment and 35 publications were included. Four additional studies were included after review of the references of already included studies. Thirty-nine manuscripts were, therefore, included in the current review and further analyzed.

Included Studies. The 39 manuscripts included in the SR and available for qualitative synthesis reported on 41 trials. General characteristics, treatments, and outcome of interest evaluated in each trial are presented in Table 1. Most manuscripts were published in peer-reviewed scientific journals. Four manuscripts were pub- 

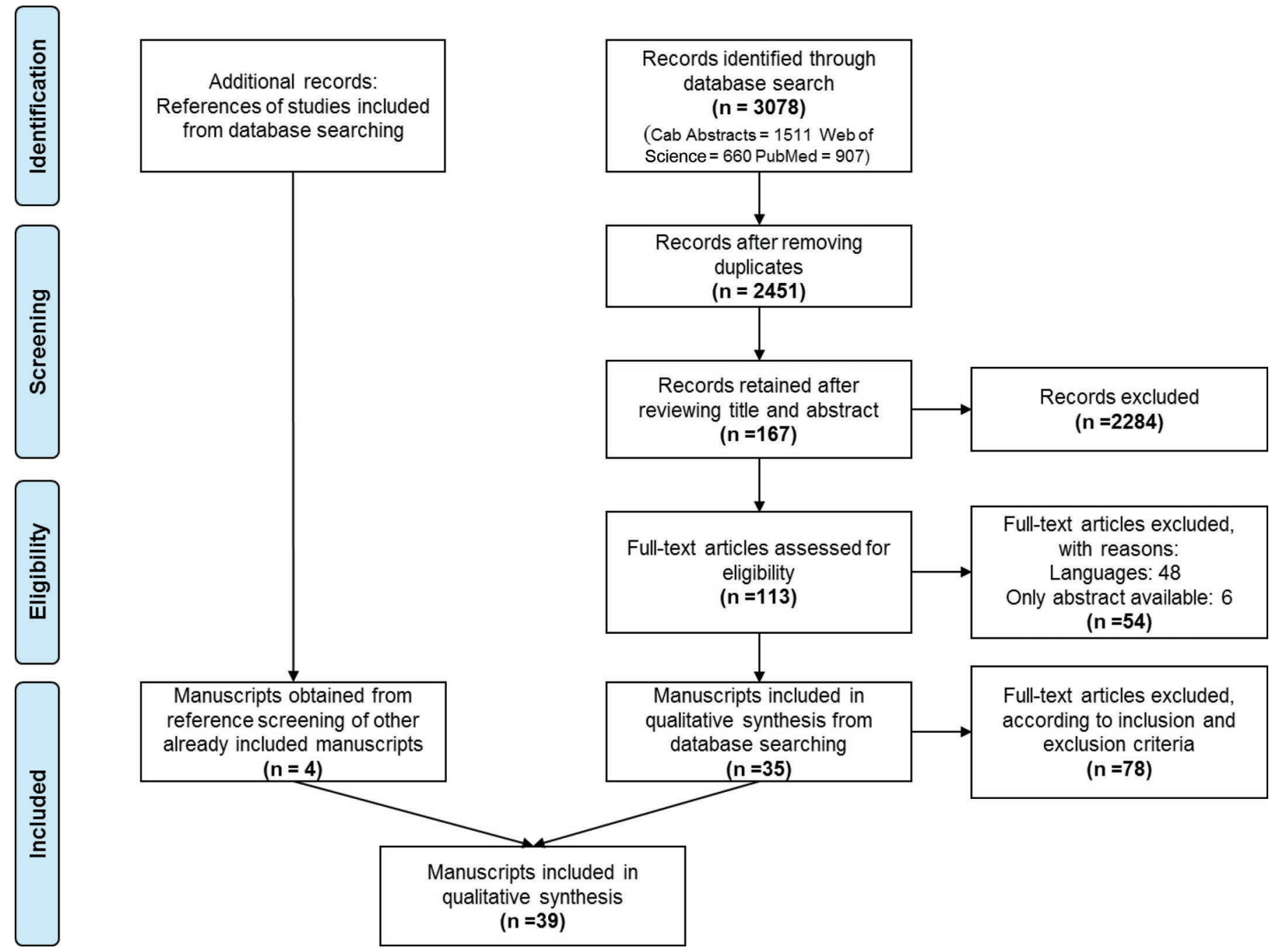

Figure 1. Result of the different steps for identifying the relevant literature in a systematic review of the literature on treatments other than conventional antimicrobials for clinical mastitis in dairy cows. Color version available online.

lished in conference proceedings (Ziv and Longo, 1991; Dinsmore et al., 1994; Elvinger et al., 1994; Mueller, 2004) and one as a thesis (Masson, 2006). Among the 41 trials, 22 were clinical trials, 18 were experimental challenges using CM models (13 using endotoxin-induced CM models, and 5 using bacteria-induced CM models), and one was an observational cohort study. The different alternative treatments evaluated in these trials were biologics $(\mathrm{n}=9)$, homeopathy $(\mathrm{n}=5)$, botanicals $(\mathrm{n}=$ $4)$, probiotics $(\mathrm{n}=2)$, and other alternative products $(\mathrm{n}=2)$. The conventional non-antimicrobial treatment investigated were oxytocin with or without frequent milking $(\mathrm{n}=5)$, and conventional anti-inflammatory drugs (nonsteroidal only: $\mathrm{n}=10$; steroidal only: $\mathrm{n}=$ 2 ; both: $\mathrm{n}=2$ ). Description of the treatment regimens evaluated, control groups used, and definitions of clinical or bacteriological cure (or both) used in each study are presented in Supplemental Table S1 (https://doi. org/10.3168/jds.2016-12512). Outcomes related to resolution of inflammation were reported in almost all trials (40/41). The effect of treatment on bacteriological cure or bacterial count was investigated in 22 trials. Finally, the effect of treatment on subsequent milk production was investigated in 15 trials.

\section{Risk of Bias in Included Studies}

The methodological quality of each of the 41 trials, including selection biases, blinding, incomplete outcome data, selective reporting, and other potential threats, is presented in Supplemental Figure S1 (https://doi. org/10.3168/jds.2016-12512). A summary of the risk of bias evaluation is presented in Figure 2. All trials had at least one potential source of bias rated as high or 


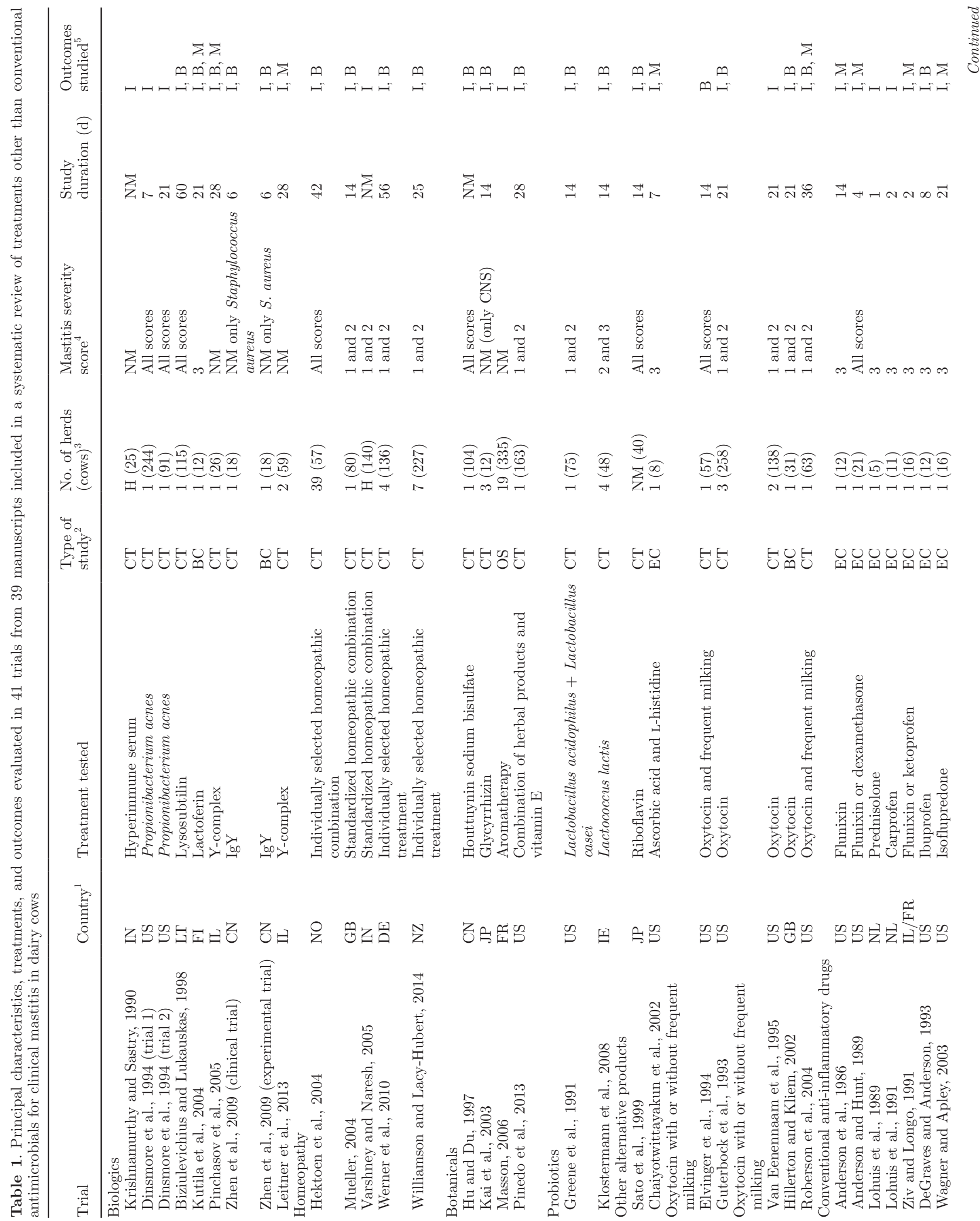


unclear. The source of bias most frequently rated as high risk were related to blinding of participants and personnel (high risk, $\mathrm{n}=17 ; 41 \%$ ), and blinding of outcome assessment (high risk $\mathrm{n}=16 ; 39 \%$ ). The most frequent reason for a high risk classification for blinding of participant and personnel was the absence of blinding and the fact that it could have influenced the care for the animals during the study. Many of these high risk studies were actually conducted on commercial dairy farms, where knowing the treatment status of a given cow is more likely to lead to a differential level of care by farm employees. Trials were classified as high risk for blinding of outcome assessment when blinding of observer was not reported and when a subjective outcome was assessed (e.g., udder or milk appearance, pain and discomfort). Selective reporting was also frequently rated as high risk (high risk: $\mathrm{n}=14 ; 34 \%$ ). In many trials no mention was made of analyses of some or many of the outcomes reported as being measured. In some other cases, an important outcome that should have been measured (given the study design used) was ignored.

In many trials $(\mathrm{n}=23 ; 56 \%)$, the risk of bias associated with random sequence generation was considered unclear either because randomization was not mentioned, or the randomization process was simply not described. Fourteen trials (34\%) were classified as having an unclear risk regarding allocation concealment based on absence of reporting on this matter in a study design in which this could have influenced the case's recruitment. In 5 trials (12\%), allocation concealment was inadequate and led to a high risk of allocation bias.

In most of the trials, animals selected were those included in the analyses or thorough explanations for exclusions were provided and nondifferential exclusion could be assumed (attrition bias, low risk: $\mathrm{n}=26$; $63 \%$ ). In only 3 trials (7\%), differential exclusion of some animals without explanations led to high risk of attrition bias.

Other potential important sources of bias for the evaluation of efficacy of non-antimicrobial treatment of CM were detected in 38 (93\%) of the trials. They included the following: the absence of a negative control group ( $\mathrm{n}=18$ trials); the absence of a positive control group ( $\mathrm{n}=17$ trials, of which 12 were an endotoxininduced CM model); the impossibility to evaluate an important outcome because of the study design used (i.e., 13 studies with endotoxin-induced $\mathrm{CM}$ and for which bacteriological cure could not be investigated); a treatment investigated or instituted in a control group that is not completely described ( $\mathrm{n}=10$ trials); inappropriate statistical analyses (2 trials); a study population that did not represent the usual commercial dairies population (2 hospital-based trials); and finally, 


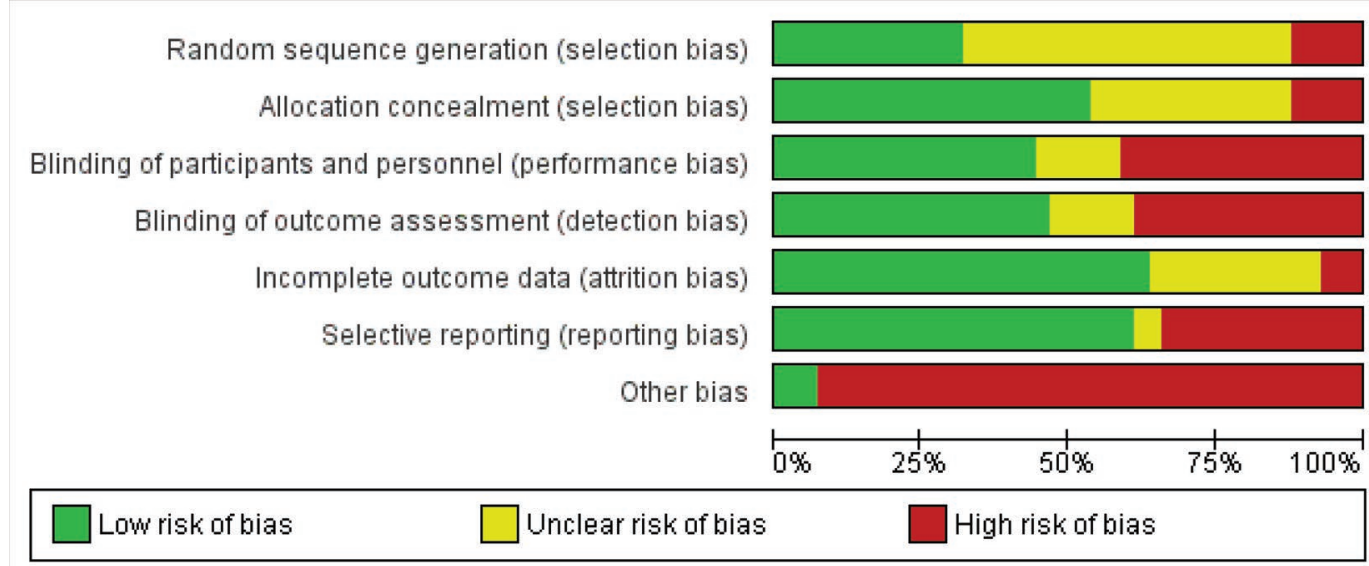

Figure 2. Proportion of studies with a given risk of bias among 41 studies included in a systematic review on treatments other than conventional antimicrobials for clinical mastitis in dairy cows. Color version available online.

a profoundly inadequate definition of bacteriological cure (1 trial). For this latter study (Klostermann et al., 2008), a bacteriological response was defined as a quarter-milk SCC $<1,000,000$ cells $/ \mathrm{mL}$ and a milk bacteriological culture yielding $<500 \mathrm{cfu} / \mathrm{mL}$ on d 14 posttreatment. According to current recommendations (Dohoo et al., 2011), such a definition would include a very large number of truly infected quarters.

Studies investigating conventional non-antimicrobial therapies had, in general, a higher number of items with low risk of bias as compared with studies investigating alternative therapies, which often had several items rated as high risk of bias or as unclear (Supplemental Figure S1; https://doi.org/10.3168/jds.2016-12512). Nevertheless, some studies investigating alternative therapies were rated as low risk of bias for most items evaluated (see Hektoen et al., 2004, as an example), illustrating that it is possible to conduct good-quality studies on alternative therapies.

\section{Effects of Interventions}

Results regarding clinical cure and bacteriological cure for the alternative and for the conventional nonantimicrobial treatments are synthesized in Tables 2 and 3, respectively. Although most studies did report on effect of treatment tested on clinical or bacteriological cure rate (or both), effect on milk production was unfrequently reported. All results reported in the 41 trials are further detailed by treatment category in the following sections.

Biologics. Three different immunoglobulin-based products were included in the study: hyper immune serum (Krishnamurthy and Sastry, 1990), Y-complex (Pinchasov et al., 2005; Leitner et al., 2013), and egg yolk immunoglobulin (Zhen et al., 2009). The authors reporting on the efficacy of the hyper immune serum observed a lower clinical improvement in the cows only treated with the hyper immune serum in comparison with those treated with antimicrobials or antimicrobials and hyper immune serum (Krishnamurthy and Sastry, 1990). Unfortunately, no statistical analyses were available. The 2 trials on the Y-complex were performed by the same research group (Pinchasov et al., 2005; Leitner et al., 2013). Results on clinical cure or bacteriological cure were not presented in these trials. Effect of treatment on milk production and on SCC was apparently investigated in Pinchasov et al. (2005); unfortunately, results of statistical analyses were not presented (Pinchasov et al., 2005). In the Leitner et al. (2013) study, comparable drops in milk yield during the $28 \mathrm{~d}$ after experimental challenge were observed between Y-complex-treated ( $30 \mathrm{~L}$ per $28 \mathrm{~d}$ ), antimicrobial-treated ( $24 \mathrm{~L}$ per $28 \mathrm{~d}$ ), and placebo-treated ( 83 L per $28 \mathrm{~d}$ ) cows. The amount of milk lost or rejected due to residue or high SCC was, however, lower in the Y-complex-treated cows (132 L per 28 d versus $277 \mathrm{~L}$ per $28 \mathrm{~d}$ in antimicrobial-treated and $280 \mathrm{~L} / 28 \mathrm{~d}$ in placebo-treated cows; $P<0.01$ ). No significance difference between treatment groups in milk conductivity or California mastitis test could be observed. Finally, the efficacy of the egg yolk immunoglobulin was tested in an experimental study as well as in a clinical trial for the treatment of CM associated solely with Staphylococcus aureus (Zhen et al., 2009). In these trials, a greater decrease occurred in bacterial count and SCC in treated cows compared with positive and negative control groups at d 6 (numbers not reported). Furthermore, in the 2 trials, the cure rates were reported to be significantly higher for immunoglobulin-treated 
cows $(3 / 6$ and $5 / 6)$ compared with both positive $(2 / 6$ and $4 / 6)$ and negative control $(0 / 6$ and $0 / 6)$ groups. For these later comparisons, however, Fisher exact tests conducted by the authors of the current review would indicate no statistical association between cure rate and treatment group. Moreover, the authors of the Zhen et al. (2009) manuscript did not report using statistical tests used to compare proportion (i.e., cure rate is indeed a proportion).

Killed extract of Propionibacterium acnes is considered as a product that is able to stimulate the immune system and was tested in 2 clinical trials and no significant difference in clinical cure was observed in comparison with the positive or negative control group in both studies (Dinsmore et al., 1994). Unfortunately, the limited data available in this later study did not support the post hoc estimation of study power.

Lysosubtilin is a preparation of lytic enzymes with antibacterial and antifungal properties (Biziulevichius and Lukauskas, 1998). It was evaluated in one clinical trial and no differences in clinical or bacteriological cures were observed when compared with positive controls (Biziulevichius and Lukauskas, 1998). Additionally, lysosubtilin at the dose of $3.5 \times 10^{6} \mathrm{IU}$ was reported to have a significantly shorter time to clinical recovery than the 2 conventional antimicrobial treatments and had a lower percentage of animals with relapse within 2 mo after treatment than the conventional antimicrobial treatment. With the exception of lysosubtilin at the dose of $3.5 \times 10^{6} \mathrm{IU}$, with a post hoc power of 78 to $85 \%$, study power was relatively low (range: $<5-37 \%$ ), thus precluding any conclusion regarding noninferiority of the lysosubtilin treatment.

Bovine lactoferrin, a glycoprotein with bacteriostatic properties, was evaluated in one experimental study (bacterial challenge) and no significant differences were observed between the treated group and the positive controls in term of resolution of clinical signs (i.e., local sign score), SCC, and milk production during the study period (i.e., up to $6.5 \mathrm{~d}$ postbacterial challenge; Kutila et al., 2004).

Homeopathy. The vast majority of trials investigating homeopathic treatments did not observe statistically significant differences in clinical or bacteriological cure between homeopathic and conventional antimicrobial treatment (i.e., the positive control group). Study power, however, was often very low (median: 12\%), and thus, these studies do not support any conclusions regarding noninferiority of the homeopathic product when compared with the conventional CM treatment. For instance, in the trial conducted by Hektoen et al. (2004), substantial differences in clinical cure rate were observed at d 7 (90\% cure in positive controls vs. $67 \%$ cure in homeopathy-treated cows), but the probability of finding a statistically significant difference, if a difference truly exists, was only $30 \%$ with the available sample size.

In 2 trials, statistically higher clinical or bacteriological cure rates for conventional treatment compared with the homeopathy-treated cows were reported [bacteriological cure rate of 83 vs. $44 \%$ at d 7 in Werner et al. (2010), and clinical cure rate of 95 vs. $63 \%$ as well as bacteriological cure rate of 74 vs. $36 \%$ at d 25 in Williamson and Lacy-Hulbert (2014)]. In the Werner et al. (2010) study, bacteriological cure rates at least 20 percentage points higher were also observed in conventionally treated compared with homeopathy-treated cows at d 14,28 , and 56 , but low study power precluded any statistical conclusion on these (Werner et al., 2010). Although cure rate in conventionally treated cows was numerically higher than that from homeopathy-treated cows on almost all evaluations, the Werner et al. (2010) study was also the only one to observe a statistically higher clinical cure rate in homeopathy-treated cows compared with conventional treatment for one of the comparisons evaluated (d 7 posttreatment; Werner et al., 2010). For that comparison, a 0\% (0/46) cure rate was observed in the positive control group. However, the statistical methods described in that manuscript (a generalized linear model with a logit function) should not allow any comparison with a group when the outcome (i.e., cure) is never observed. A Fisher exact test conducted by the authors of the current review did indicate, however, that this was indeed a significant difference.

One other study showed a numerically higher cure rate in the homeopathy-treated cows (87\%) compared with the positive controls (59\%; Varshney and Naresh, 2005). No statistical analyses were presented in this later study, but a chi-squared test using the raw results and conducted by the authors of the current review yielded a $P$-value $<0.01$. In the Varshney and Naresh (2005) study, however, animals that did not respond to the homeopathic treatment within a few days were removed from the study, which is a very important source of bias.

When compared with placebo or untreated cows, in all instances no difference in clinical or bacteriological cure was observed in homeopathy-treated cows, with the exception of one study where a higher cure rate at $d$ 56 posttreatment was observed in homeopathy-treated cows (36 vs. 16\%; Werner et al., 2010). These investigators, however, were not able to demonstrate cure rate differences between negative controls and homeopathytreated cows at any other evaluation time (i.e., on d 7 , 14, and 28).

Observations other than general clinical or bacteriological cure rates were also reported in these studies. 
Table 2. Proportion of clinically (CC) or bacteriologically (BC) cured animals in treated and control groups and study power for the 13 trials from 12 manuscripts evaluating an alternative therapy (i.e., alternative to the mainstream allopathic medicine) and included in a systematic review of treatments other than conventional antimicrobials for clinical mastitis in dairy cows

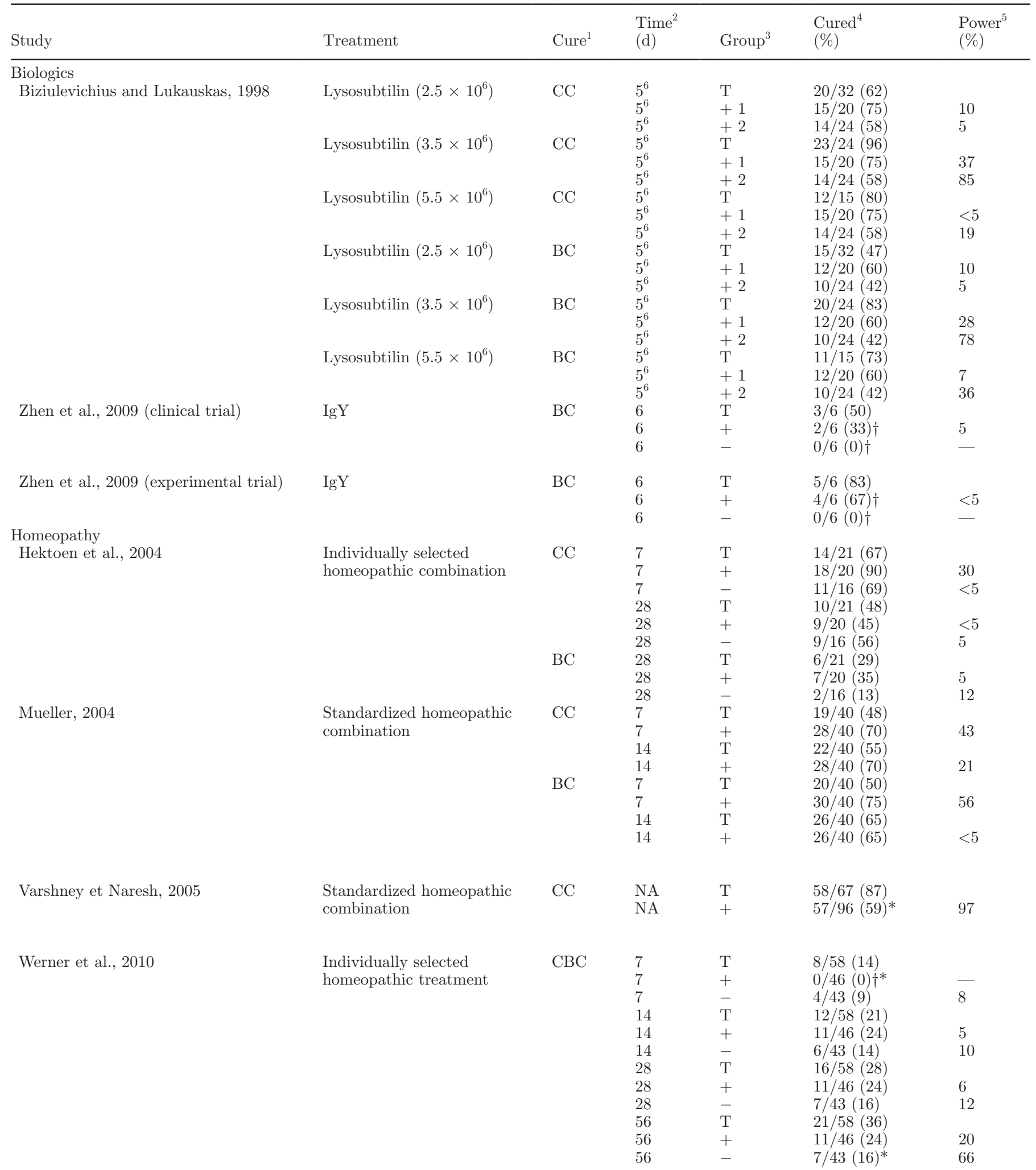


Table 2 (Continued). Proportion of clinically (CC) or bacteriologically (BC) cured animals in treated and control groups and study power for the 13 trials from 12 manuscripts evaluating an alternative therapy (i.e., alternative to the mainstream allopathic medicine) and included in a systematic review of treatments other than conventional antimicrobials for clinical mastitis in dairy cows

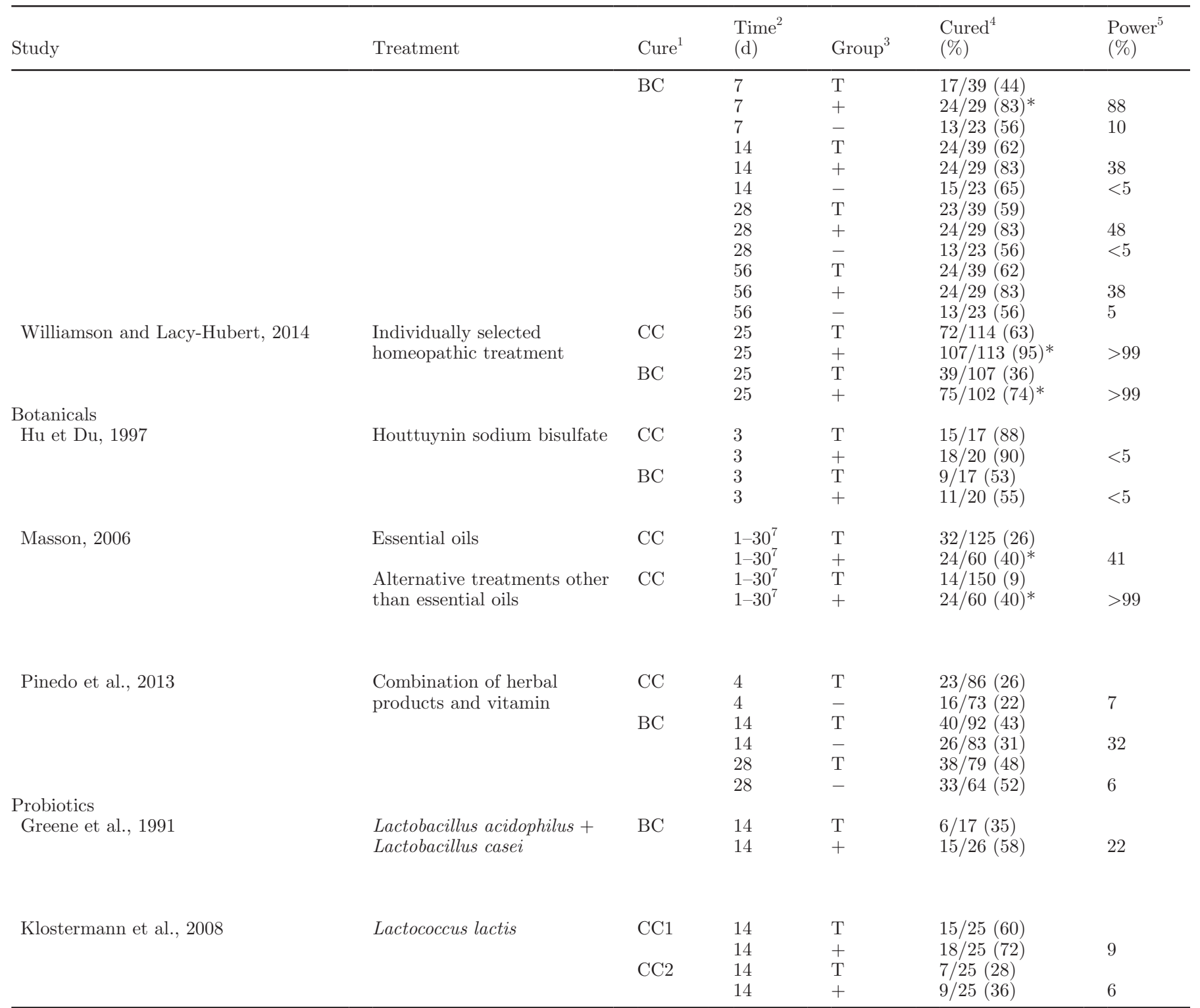

${ }^{1}$ Outcome studied: clinical cure $(\mathrm{CC})$, bacteriological cure $(\mathrm{BC})$, mixture of clinical and bacteriological cure $(\mathrm{CBC})$. CC1 = clinical cure of the first trial; $\mathrm{CC} 2=$ clinical cure of the second trial.

${ }^{2}$ Number of days after instigation of treatment when outcome was evaluated. NA $=$ not applicable.

${ }^{3}$ Group studied: treated $(\mathrm{T})$; positive controls $(+)$; negative controls $(-) .+1=$ first positive control; $+2=$ second positive control.

${ }^{4}$ Proportion of animals (or quarters) cured.

${ }^{5}$ Power of the study to conclude on statistical significance regarding the observed difference between treatment and positive control group or between treatment and negative control group given the sample size chosen and using an $\alpha$ threshold of 0.05.

${ }^{6}$ Described as up to $5 \mathrm{~d}$.

${ }^{7}$ Measured at first milk control following the clinical case.

$\dagger$ Statistical methods reported seemed inappropriate and authors of the current review replaced it with a Fisher exact test; the result of this later test is reported.

*Asterisks indicate, for a given outcome, a statistically significant difference in proportion of cure when compared with treatment under investigation $(P<0.05)$. 
Table 3. Proportion of cured animals in treated and control groups and study power for the 5 trials from 5 manuscripts evaluating nonantimicrobial conventional therapies and included in a systematic review of treatments other than conventional antimicrobials for clinical mastitis in dairy cows

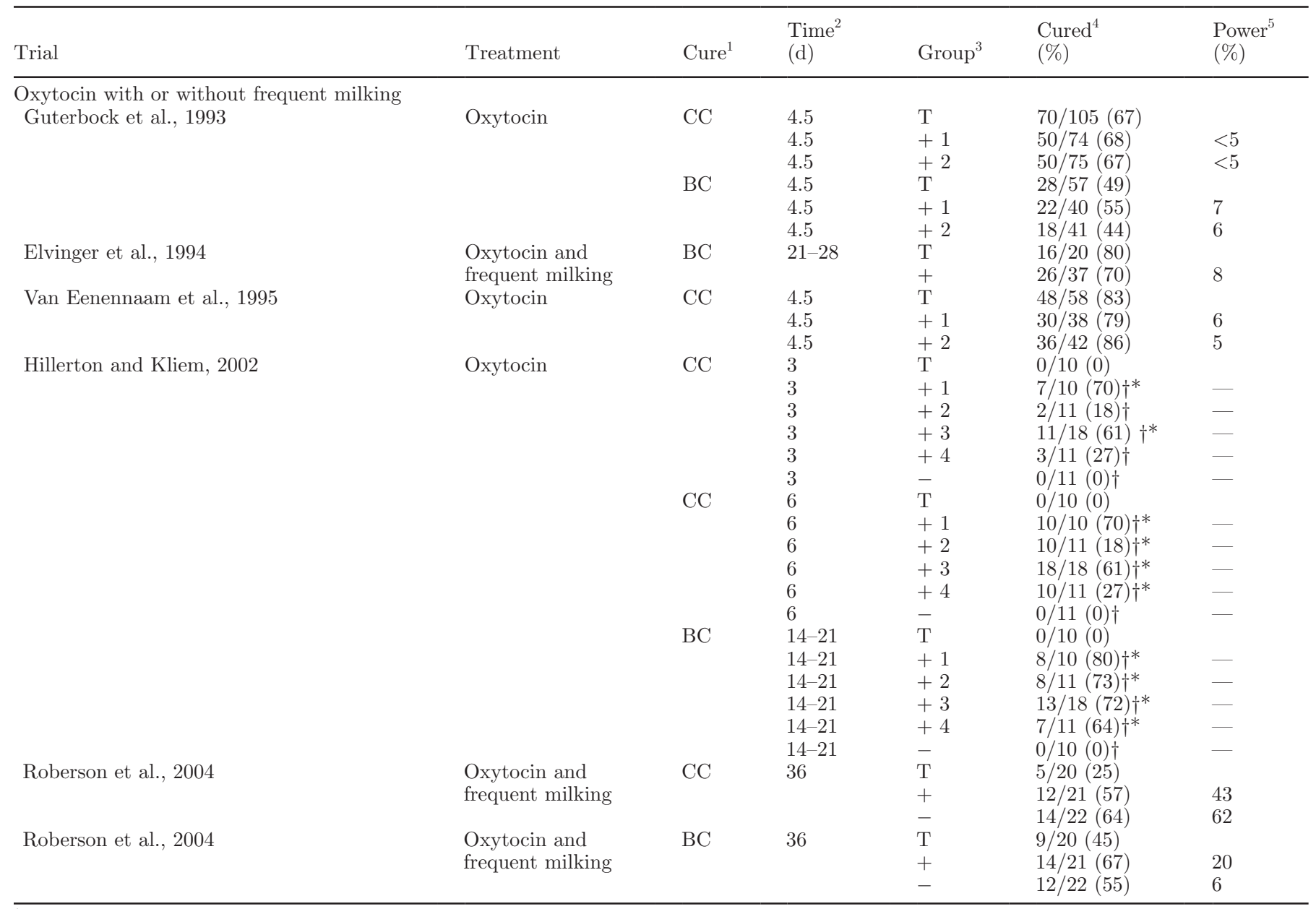

${ }^{1}$ Outcome studied: clinical cure (CC), bacteriological cure (BC).

${ }^{2}$ Number of days after instigation of treatment when outcome was evaluated.

${ }^{3}$ Group studied: treated $(\mathrm{T})$; positive controls $(+)$; negative controls $(-) .+1=$ first positive control; $+2=$ second positive control; $+3=$ third positive control; $+4=$ fourth positive control.

${ }^{4}$ Proportion of animals (or quarters) cured.

${ }^{5}$ Power of the study to conclude on statistical significance regarding the observed difference between treatment and positive control group or between treatment and negative control group given the sample size chosen and using an $\alpha$ threshold of 0.05 .

†Statistical methods reported seemed inappropriate and authors of the current review replaced it with a Fisher exact test; in this case, results of this later test are reported.

*Asterisks indicate, for a given outcome, a statistically significant difference in proportion of cure when compared with treatment under investigation $(P<0.05)$.

Mueller (2004) reported no difference in CM recurrence rate or in number of cows exceeding 200,000 cells/mL at next DHI test between cows treated with ampicillin IM or homeopathy, but exact numbers were not provided (Mueller, 2004). Varshney and Naresh (2005) reported a significantly longer mean recovery period in homeopathic-treated cows $(7.7$ vs. $4.5 \mathrm{~d}$ in antimicrobial-treated cows; $P<0.05$ ), but significantly lower mean treatment costs in homeopathy-treated cows (US $\$ 0.47$ vs. US $\$ 33 ; P<0.05$ ). Finally, William- son and Lacy-Hulbert (2014) reported that, for IMI caused by Streptococcus uberis, a significantly higher bacteriological cure rate was achieve in antimicrobialtreated cows $(48 / 65 ; 74 \%)$ compared with cows treated with homeopathic remedies $(24 / 75 ; 32 \%)$. None of the studies investigating homeopathic treatment evaluated the effect of treatment on milk yield.

Botanicals. The different phytotherapeutic products included in the study were combination of essential oils (Masson, 2006; Pinedo et al., 2013), houttuynin 
sodium bisulfate (Hu and $\mathrm{Du}, 1997)$, and glycyrrhizin (Kai et al., 2003). Three trials reported specific data on clinical or bacteriological cure (or both). In 2 of these studies (Hu and Du, 1997; Pinedo et al., 2013), however, study power was often very low (median: 12\%), and thus, these studies do not support any conclusions regarding noninferiority or nonsuperiority of these botanicals treatments. In the $\mathrm{Hu}$ and $\mathrm{Du}$ (1997) trial, very similar and high cure rates were observed between conventional and alternative treatments (88 vs. 90\%). In Masson (2006), significantly higher cure rates were observed in the conventionally treated cases compared with the cows treated with essential oils [odds ratio $(\mathbf{O R}): 2.0 ; P=0.01]$ or to the cows treated with other alternative treatments (OR: $2.5 ; P=0.01$ ). In the Pinedo et al. (2013) study, no differences in clinical or bacteriological cure rates were observed between cows treated with essential oils and a negative control group. Additionally, no significant differences were observed between cows treated with essential oils and cows receiving no treatment in terms of hazard of recovery and subsequent SCS. However, a shorter time to recovery was reported in cows treated with essential oils (4.6 vs. $6.5 \mathrm{~d}$ in untreated animals), a significantly larger SCS reduction was also reported in cows treated with essential oils (numbers not presented; $P=0.04$ ).

Finally, Kai et al. (2003) observed differences in udder firmness and in gland swelling between glycyrrhizintreated and antimicrobial-treated cows (i.e., lower firmness score at d 2 and 7 for glycyrrhizin-treated cows, but higher swelling score at d 1, 2, and 7). They also observed lower coagulase-negative staphylococci count in milk of antimicrobial-treated cows at d 1, 2, and 7 . None of the trials investigating botanicals investigated effect of treatment on milk yield.

Probiotics. A combination of Lactobacillus casei and Lactobacillus acidophilus (Greene et al., 1991) and Lactococcus lactis DPC3174 (Klostermann et al., 2008) were the 2 probiotics tested in 2 different clinical trials. No differences were found in bacteriological or clinical cure rates between the intramammary probiotic treatment and the intramammary antimicrobial treatment. In all cases, however, numerically higher cure rates were observed in the antimicrobial-treated cows. Study power, however, was relatively low (range: 6-22\%), thus precluding any conclusion regarding noninferiority of the probiotic treatment. In one study the SCC was significantly higher at $\mathrm{d} 7$ and 14 in the probiotic group, but no SCC differences could be observed 2 to 3 mo after treatment (Greene et al., 1991).

Vitamins. Vitamin $\mathrm{B}_{2}$ (Sato et al., 1999) and vitamin $\mathrm{C}$ in combination or not with L-histidine (Chaiyotwittayakun et al., 2002) were the different vitamins tested for their properties to enhance the immune system. Vitamin $\mathrm{B}_{2}$ was tested in one clinical trial, and although lower SCC at d 3, 7, and 14 were observed, no difference in bacterial count was observed when compared with negative controls (Sato et al., 1999). The efficacy of vitamin $\mathrm{C}$ and L-histidine, alone or in combination, was evaluated in an endotoxin experimental study (Chaiyotwittayakun et al., 2002). For both of these molecules, no significant differences in clinical signs resolution (e.g., heart rate, respiratory rate, rectal temperature, ruminal contraction rate, DMI) were observed in comparison with no treatment. Additionally, no differences were observed in quarter milk production for both molecules, whereas a significantly higher composite (i.e., sum of the 4 quarters) milk production was observed for ascorbic acid between d 3 and 5 of the experiment.

Oxytocin With or Without Frequent Milk Out. Five trials (4 clinical trials and 1 experimental study) evaluated the effect of oxytocin injection; 2 trials evaluated oxytocin with additional milking and 3 without additional milking. Overall results showed similar or lower bacteriological and clinical cure rates for oxytocin treatment with or without frequent milking compared with antimicrobial treatment (Guterbock et al., 1993; Elvinger et al., 1994; Van Eenennaam et al., 1995; Roberson et al., 2004). Again, the power of these studies was relatively low (median: 6\%) and the fact that cure rate in oxytocin-treated animals was not different than that of positive controls should not be interpreted as a statement of noninferiority of the oxytocin treatment. Two studies also compared administration of oxytocin to no treatment; in both cases, the oxytocin-treated cows had clinical and bacteriological cure rates comparable to the untreated cows (Hillerton and Kliem, 2002; Roberson et al., 2004).

In addition to clinical cure rate comparisons, Van Eenennaam et al. (1995) also reported a significantly greater proportion of relapse (i.e., retreatment or relapse of $\mathrm{CM}$ within $21 \mathrm{~d}$ of the initial case; 18/58 vs. 5/42) and of $\mathrm{CM}$ in another mammary quarter (16/58 vs. 4/42) in oxytocin-treated compared with cephapirin-treated cows (Van Eenennaam et al., 1995). No differences were observed, however, in CM recurrence (i.e., CM event in the same quarter $>21 \mathrm{~d}$ after initial case; $7 / 58$ in the oxytocin group vs. $8 / 42$ in the cephapirin group). Significantly lower costs were, however, reported for the oxytocin-treated cows (US\$34.88) compared with cephapirin-treated cows (US\$54.47). No significant differences in daily milk or 305-d milk production, time to return to normal secretion, number of nonsalable milkings, or time to culling could be highlighted between oxytocin-treated and positive controls. 
In the experimental Streptococcus uberis model study, oxytocin had significantly lower clinical cure rate and bacteriological cure rate over antimicrobial treatment alone (Hillerton and Kliem, 2002). Finally, in the Roberson et al. (2004) study, in addition to general cure rate comparisons, detrimental effects on bacteriological cure were reported for specific pathogens, more particularly Streptococcus spp. Moreover, in that study, animals frequently milked out had a significantly higher rate of high microbial growth (i.e., $>700 \mathrm{cfu} / \mathrm{mL}$ ) within the first $3 \mathrm{~d}$ of treatment than the antimicrobial-treated and untreated groups (Roberson et al., 2004). Additionally, no differences with untreated cows were observed in this study in term of milk production or development of IMI in co-quarters (Roberson et al., 2004).

Anti-Inflammatory Drugs. Four nonsteroidal anti-inflammatory drugs (NSAID) and 3 steroidal anti-inflammatory drugs (SAID) were evaluated in 14 experimental studies of CM using either an endotoxin $(\mathrm{n}=12)$ or bacterial challenge $(\mathrm{n}=2)$ model. The NSAID included were flunixin meglumine (8 trials), carprofen (2 trials), ketoprofen (2 trials), and ibuprofen (1 trial). The SAID tested were isoflupredone acetate (2 trials), dexamethasone (1 trial), and prednisolone ( 1 trial). Due to the study design used in most studies (i.e., endotoxin challenge) clinical and bacteriological cure rates could not be investigated.

Unsurprisingly, the most consistent clinical effect observed in these studies was a significant decrease in body temperature in comparison with no treatment, with the exception of the 2 trials on isoflupredone acetate (Wagner and Apley, 2003, 2004) and 1 trial on flunixine meglumine (Anderson and Hunt, 1989), which did not observe a difference in body temperature between treated and untreated cows. Milk modification based on clinical aspect, SCC, or milk conductivity was not affected by administration of anti-inflammatory drugs with the exception of 1 trial on carprofen in which milk modification was worsened in comparison with no treatment, and 1 trial on ketoprofen in which it was improved. For the 2 Escherichia coli induced CM model (flunixin meglumine and carprofen), no difference was observed with the no-treatment group in term of resolution of clinical signs (Vangroenweghe et al., 2005; Yeiser et al., 2012).

In the 2 studies using bacterial E. coli challenges, no differences were observed in term of bacteriological counts in comparison with no treatment (Vangroenweghe et al., 2005; Yeiser et al., 2012). In general, no difference on milk production was observed in comparison with no treatment with the exception of 1 study on dexamethasone in which milk production was lower (Anderson and Hunt, 1989) and 1 study on carprofen in which it was higher (Yeiser et al., 2012).

\section{DISCUSSION}

\section{Summary of the Main Results}

Mastitis control and treatment is the main cause of antimicrobial drugs use in dairy herds. In a context of a reduction or nonutilization of antimicrobial drugs, treatments other than conventional antimicrobial treatments that have already proven efficiency must be identified. A large number of alternative measures are available and have already been studied. However, one of the most valuable results of this SR was, perhaps, the appraisal of the very low number of studies without high risk of bias, as well as the limited statistical power of these studies. The validity of many of the included studies is consequently questionable. This prevents us from corroborating the results of these studies, and consequently, drawing definitive conclusions of the efficiency of these treatments is almost impossible. On the other hand, consistency in the absence of positive results, as well as reported detrimental effects, support the recommendation of not using some nonantimicrobial treatments such as homeopathy, oxytocin with or without frequent milking out, and probiotics.

\section{Overall Completeness and Applicability of Evidence}

The main goal of this SR was to identify treatments other than conventional antimicrobials that could be effective for the treatment of CM in dairy cows based on clinical signs resolution, bacteriological cure, and milk production. Unfortunately, milk production was one of the most frequently underreported outcome. Because it is an important outcome, particularly in term of costeffectiveness evaluation, this could be a limitation for application in practice.

Two of the included clinical trials were performed in an hospital setting [1 homeopathy (Varshney and Naresh, 2005) and 1 biologic (Krishnamurthy and Sastry, 1990)]. This raises the question of applicability in commercial dairies. Additionally, many experimental studies were retained in the current SR. However, one could argue that they cannot fully represent CM as observed in commercial dairies. This is particularly true for endotoxin-induced mastitis. Efficacy of conventional anti-inflammatory drugs alone was only evaluated in experimental models of $\mathrm{CM}$, making conclusions on their efficacy in field conditions impossible.

One important limitation for applicability of the results of this study in the field is the lack of availability of many products. For example, to our knowledge, lysosubtilin or immune-based products such a Y-complex or egg-complex are not commercially available in North America. 
Additionally, one should take into account that in most of the trials included in this SR, these treatments were evaluated in monotherapy. In general, the goal of the alternative approaches is to act at different levels at the same time in the treatment of infection: (1) direct killing of the microbial (antimicrobial properties), (2) stimulation of the immune system (immunomodulation properties), and (3) control of inflammatory processes and associated signs (anti-inflammatory properties; Karreman, 2009). For these reasons, different substances often need to be combined in a multi-pronged approach.

Finally, due to the small number of studies reporting on a given treatment, to the different negative and positive control groups used, and to the various bacteriological and clinical cure definitions used, integrating the results from different studies in a meta-analysis was not possible. With a larger number of studies conducted and reported in a more homogeneous manner on a given treatment, an overall estimate of effect could have been computed for some therapies.

\section{Quality of the Evidence}

As mentioned previously, one major issue of this SR was the very low number of studies without high risk of bias. Indeed, apart from 1 publication that did not report sufficiently to fully assess risk of bias (Zhen et al., 2009), all of the 40 remaining trials selected had at least 1 high risk of bias on the 7 sources of bias evaluated. Various aspects of the included studies, from conception through implementation and up to reporting may thus have affected the quality of the evidence. Moreover, studies investigating alternative treatments often were scored as high risk of bias on many items. The poorer quality of these studies seemed to be due to poor study planning, poor implementation, and lack of knowledge on $\mathrm{RCT}$, rather than to a difficulty inherent to the therapies investigated. Indeed, some authors were able to conduct very good quality studies on alternative products (Supplemental Figure S1; https:// doi.org/10.3168/jds.2016-12512). Given the financial resources that were invested in all these studies on alternative therapies, a minimal recommendation would be to use the Consolidated Standards of Reporting Trials (CONSORT) statement (www.consort-statement. org) to guide study planning and to incorporate to the research team a scientist with knowledge on RCT planning and implementation.

Furthermore, most studies investigating alternative treatments were directly tested in clinical trials conducted on commercial dairies without any prior efficacy investigation in an experimental model of CM. Conducting a RCT is, indeed, necessary to support adoption of a novel treatment. Recently, Gomes and Henriques (2016) reviewed the therapeutic approach for bovine mastitis and concluded that despite encouraging in vitro studies, in vivo studies are still required before drawing any conclusions. Some precautions are, however, necessary before testing a novel therapy on commercial dairies. Furthermore, previous demonstration of a treatment's biological relevance (e.g., an in vitro study, a proof of concept) is essential for correct interpretation of the results of a subsequent RCT. The fact that studies reporting on biological relevance are lacking for most alternative treatments is very disconcerting.

On the other hand, efficacy of conventional antiinflammatory drugs alone was only evaluated in CM experimental models. These drugs have demonstrated potential beneficial effects mainly on control of clinical signs. Whether these drugs can be beneficial as part of a CM treatment should now be evaluated using RCT.

Another common feature of all included studies is the small number using both negative and positive control groups in their protocol. Only 6/41 studies presented comparisons between a treatment group and positive and negative control groups (Supplemental Table S1; https://doi.org/10.3168/jds.2016-12512, Table 2, and Table 3). Without both control groups, it is impossible to fully study the intrinsic efficiency of the alternative treatment. Furthermore, study designs comparing a novel approach with a conventional one (i.e., using a positive control group) require the very strict application of some core principles including a priori determination of the noninferiority margin, and a priori sample size or power determination (or both). Noninferiority margin was not discussed in any of the included articles using positive controls and a priori sample size determination or power was only reported in 2 studies (Guterbock et al., 1993; Hektoen et al., 2004).

\section{Potential Biases in the Review Process}

Language restriction is a common bias to SR or meta-analysis. This barrier prevented the authors of the current review from having a general overview of all publications. In the current SR, 42\% (48/113) of potentially relevant publications were excluded due to language restriction without a complete analysis. Among these, publications in Russian, German, and Chinese were among the most frequent. It was reported that language restriction (i.e., inclusion of only English published trials) underestimates the effectiveness of alternative treatments (Pham et al., 2005). Additionally, Chinese biomedical databases were not searched in the present study, which may have led to underidentification of clinical evidence for alternative treat- 
ments (Cohen et al., 2015). For instance, no article analyzing the effect of acupuncture on CM was retained because the 4 potential manuscripts of interest were in Russian, Chinese, and German. As stated by Cohen et al. (2015), this underlines the necessity for increasing accessibility to Chinese databases and for collaboration between English-speaking and non-English-speaking research groups (Cohen et al., 2015).

\section{Agreement and Disagreements with Other Studies or Reviews}

To our knowledge, no SR on the efficacy of treatments other than conventional antimicrobials for CM is available. However, 2 previous published reviews concluded that it was impossible to make any real conclusions on therapeutic efficiency of alternative veterinary products for the treatment of $\mathrm{CM}$ due to insufficient available data (Ruegg, 2009; Ameloot, 2010). The current SR arrives more or less at the same conclusions. In addition, common flaws and pitfalls were identified in the current review and conclusions could be drawn regarding nonefficacy, and in some instances, problematic side-effects of homeopathy, oxytocin with or without frequent milk out, and intramammary infusion of probiotics.

Different SR or meta-analyses on homeopathy have already been published in veterinary medicine (Mathie and Clausen, 2014, 2015a,b; Mathie et al., 2016). One major concern reported in all these studies is the overall poor quality of trials found. According to the authors, the low number of high-quality studies precludes any definitive conclusions on the effectiveness of homeopathy (Mathie and Clausen, 2014, 2015b). Despite this, the conclusion of these studies is that, according to the data actually available, very little evidence is available to indicate that individualized homeopathy treatment could be distinguished from placebo. In some cases relatively small, specific effects could be observed (Mathie and Clausen, 2015b; Mathie et al., 2016); however, these small effects originated mainly from prophylaxis trials (Mathie and Clausen, 2015b). However, these little effects originated mainly from prophylaxis trials (Mathie and Clausen, 2015a). Additionally, Mathie et Clausen (2014) reported that the efficacy of individualized homeopathic treatment was not different from placebo treatment for bovine mastitis (Mathie and Clausen, 2014).

Two SR have been performed to identify medicinal plants that could have potential beneficial effects in veterinary medicine (Mayer et al., 2014; Ayrle et al., 2016). Both SR concluded that botanical products need further in vivo investigations and particularly clinical trials before any conclusions can be drawn on their efficacy. Mayer et al. (2014) concluded that the European ethnoveterinary medicine offers a solid basis for stimulating research in veterinary phytotherapy in Europe with a perspective to solve animal health problems in organic or even nonorganic ruminant production. Results seem thus promising, but studies require more rigors and constants. Mallow and St. John's wort were the 2 plants identified to have potential beneficial effect as topical agents in cases of mastitis (Mayer et al., 2014).

Roberson (2012), in his review on the treatment of $\mathrm{CM}$, reported that anti-inflammatory drugs must be considered in the treatment of CM. However, he also stated that many of the studies evaluating anti-inflammatory drugs were experimental studies and that no consensus was available on the beneficial effect of these drugs. Additionally, it is most likely that these will not alter the outcome of grade 1 and $2 \mathrm{CM}$ (Roberson, 2012). As mentioned previously, because no clinical trials are available for the evaluation of anti-inflammatory drugs in field condition, their efficacy cannot be determined.

It is often mentioned and seems intuitive that the mammary gland health could be improved with more frequent milk out with or without concurrent use of oxytocin. Indeed this would potentially lead to removal of bacteria from the mammary gland and, therefore, facilitate cure. The current SR concluded that the efficacy of oxytocin with or without frequent milking out alone is inconsistent and some potential detrimental effects were reported. However, in one study on frequent milk out used in association with antimicrobial treatment or NSAID, a positive effect on clinical cure was observed (Suojala et al., 2010).

Finally, few alternative therapies are approved for use in dairy cows by regulatory agencies. Although some are approved as food additives, information is currently lacking regarding their safety for cows and, ultimately, for consumers. This lack of animal and food safety evidence, combined with the fact that their efficacy was not demonstrated so far, should deter us from using alternative therapies on commercial dairies.

\section{Implications for Research}

This SR clearly underlines the urgent need for welldesigned randomized clinical trials for the evaluation of alternative treatment for CM. Ultimately, researchers must prove the efficacy of the alternative treatment and first confirm their potential safety (for the cows and the consumers) and efficacy in experimental models. Additionally, the role of conventional anti-inflammatory drugs needs to be confirmed by RCT. Finally, to minimize bias, using the CONSORT statement, not only for reporting, but also for designing clinical trials, is strongly encouraged. 


\section{CONCLUSIONS}

Unfortunately, because of the low number of highquality trials for each treatment, no evidence-based recommendations can be given for the use of alternative or non-antimicrobial conventional treatment for CM. However, because of reported detrimental effects and lack of evidence of an effect on bacteriological or clinical cure rate, probiotics and oxytocin with or without frequent milk out are not recommended. Additionally, results from this SR would support a conclusion toward nonefficacy of homeopathic treatments for CM treatment.

\section{ACKNOWLEDGMENTS}

The authors acknowledge Huguette Malet, librarian, Faculté de médecine vétérinaire, Université de Montréal, Canada, for her valuable help. This research is funded by the AgriInnovation Program of Agriculture and Agri-Food Canada's Growing Forward 2 Policy Framework (a federal-provincial-territorial initiative), Dairy Farmers of Canada, and Valacta Inc. through the Organic Science Cluster II, an industry-supported research and development endeavor initiated by the Organic Agriculture Centre of Canada at Dalhousie University in collaboration with the Organic Federation of Canada. DF is the guarantor of the current review. $\mathrm{DF}, \mathrm{VW}, \mathrm{JPD}$, and SD contributed to establishing the protocol for the systematic review. DF, VW, JPD, and SD were responsible for selecting articles, extracting data, and analyzing them. JPR, PL, SD, and FL provided their expertise on IMI and organic dairy farming. All authors reviewed and provided feedback on the manuscript.

\section{REFERENCES}

Agriculture and Agri-Food Canada. 2012. Organic Dairy Industry in Canada-2012 Edition. Agriculture and Agri-Food Canada.

Ameloot, C. 2010. Thérapeutique alternatives des mammites. Soigner les mammites autrement. Bull. Group. Tech. Vet. 54:69-75.

Anderson, K. L., and E. Hunt. 1989. Anti-inflammatory therapy in acute endotoxin-induced bovine mastitis. Vet. Res. Commun. $13: 17-26$.

Anderson, K. L., A. R. Smith, R. D. Shanks, L. E. Davis, and B. K. Gustafsson. 1986. Efficacy of flunixin meglumine for the treatment of endotoxin-induced bovine mastitis. Am. J. Vet. Res. 47:13661372 .

Ayrle, H., M. Mevissen, M. Kaske, H. Nathues, N. Gruetzner, M. Melzig, and M. Walkenhorst. 2016. Medicinal plants-Prophylactic and therapeutic options for gastrointestinal and respiratory diseases in calves and piglets? A systematic review. BMC Vet. Res. $12: 89$.

Banting, A., S. Banting, K. Heinonen, and K. Mustonen. 2008. Efficacy of oral and parenteral ketoprofen in lactating cows with endotoxin-induced acute mastitis. Vet. Rec. 163:506-509.
Biziulevichius, G. A., and K. Lukauskas. 1998. In vivo studies on lysosubtilin. 3. Efficacy for treatment of mastitis and superficial lesions of the udder and teats in cows. Vet. Res. 29:441-456.

Chaiyotwittayakun, A., R. J. Erskine, P. C. Bartlett, T. H. Herd, P. M. Sears, and R. J. Harmont. 2002. The effect of ascorbic acid and L-histidine therapy on acute mammary inflammation in dairy cattle. J. Dairy Sci. 85:60-67.

Chapinal, N., C. E. Fitzpatrick, K. E. Leslie, and S. A. Wagner. 2013. Experimentally induced mastitis reduces weight shifting between the rear legs while standing in dairy cows. J. Dairy Sci. 96:30393043.

Chapinal, N., C. E. Fitzpatrick, K. E. Leslie, and S. A. Wagner. 2014. Short communication: Automated assessment of the effect of flunixin meglumine on rumination in dairy cows with endotoxin-induced mastitis. Can. J. Anim. Sci. 94:21-25.

Cohen, J. F., D. A. Korevaar, J. Wang, R. Spijker, and P. M. Bossuyt. 2015. Should we search Chinese biomedical databases when performing systematic reviews? Syst. Rev. 4:23.

DeGraves, F. J., and K. L. Anderson. 1993. Ibuprofen treatment of endotoxin-induced mastitis in cows. Am. J. Vet. Res. 54:1128-1132.

Dinsmore, R. P., M. D. Salman, and M. B. Cattell. 1994. Field trial of the use of Propionibacterium acnes immunostimulant in the treatment of acute clinical mastitis. Page 183 in Proc. Annual Conf. Am. Assoc. Bov. Pract. The Association 1994-1995, Stillwater, OK.

Dohoo, I. R., J. Smith, S. Andersen, D. F. Kelton, and S. Godden., and Mastitis Research Workers' Conference. 2011. Diagnosing intramammary infections: Evaluation of definitions based on a single milk sample. J. Dairy Sci. 94:250-261.

Dufour, S., H. W. Barkema, L. DesCoteaux, T. J. DeVries, I. R. Dohoo, K. Reyher, J. P. Roy, and D. T. Scholl. 2010. Development and validation of a bilingual questionnaire for measuring udder health related management practices on dairy farms. Prev. Vet. Med. 95:74-85.

Elvinger, F., C. K. Watson, J. R. Cole, and J. W. West. 1994. Use or non-use of antibiotics in clinical mastitis cases. Pages 379-380 in Proc. 33rd Natl. Mastitis Counc. Annual Meet. Natl. Mastitis Council, Orlando, FL. National Mastitis Council, New Prague, $\mathrm{MN}$.

Francoz, D., G. Fecteau, G. Côté, O. Labrecque, C. A. Gagnon, and F. Ramanantoanini. 2011. Évaluation de la prévalence individuelle et de troupeau de 4 maladies d'importance et des facteurs de risques associés à la présence de ces maladies dans les troupeaux laitiers biologiques du Québec. Page 61 in Programme de soutien au développement de l'agriculture biologique, Saint-Hyacinthe, QC, Canada.

Gomes, F., and M. Henriques. 2016. Control of bovine mastitis: Old and recent therapeutic approaches. Curr. Microbiol. 72:377-382.

Greene, W. A., A. M. Gano, K. L. Smith, J. S. Hogan, and D. A. Todhunter. 1991. Comparison of probiotic and antibiotic intramammary therapy of cattle with clinical mastitis. Bov. Pract. 26:119-122.

Guterbock, W. M., A. L. v. Eenennaam, R. J. Anderson, I. A. Gardner, J. S. Cullor, and C. A. Holmberg. 1993. Efficacy of intramammary antibiotic therapy for treatment of clinical mastitis caused by environmental pathogens. J. Dairy Sci. 76:3437-3444.

Halasa, T., K. Huijps, O. Osteras, and H. Hogeveen. 2007. Economic effects of bovine mastitis and mastitis management: A review. Vet. Q. 29:18-31.

Hektoen, L., S. Larsen, S. A. Odegaard, and T. Loken. 2004. Comparison of homeopathy, placebo and antibiotic treatment of clinical mastitis in dairy cows - Methodological issues and results from a randomized-clinical trial. J. Vet. Med. A 51:439-446.

Hillerton, J. E., and K. E. Kliem. 2002. Effective treatment of Streptococcus uberis clinical mastitis to minimize the use of antibiotics. J. Dairy Sci. 85:1009-1014.

Hu, S. H., and A. F. Du. 1997. Treatment of bovine mastitis with houttuynin sodium bisulphate. J. Vet. Med. B 44:365-370.

Kai, K., K. Komine, K. Asai, T. Kuroishi, Y. Komine, T. Kozutsumi, M. Itagaki, M. Ohta, Y. Endo, and K. Kumagai. 2003. Anti-inflammatory effects of intramammary infusions of glycyrrhizin in 
lactating cows with mastitis caused by coagulase-negative staphylococci. Am. J. Vet. Res. 64:1213-1220.

Karreman, H. J. 2009. Disease control on organic and natural cattle operations. Anim. Health Res. Rev. 10:121-124.

Klostermann, K., F. Crispie, J. Flynn, R. P. Ross, C. Hill, and W. Meaney. 2008. Intramammary infusion of a live culture of Lactococcus lactis for treatment of bovine mastitis: Comparison with antibiotic treatment in field trials. J. Dairy Res. 75:365-373.

Krishnamurthy, M., and K. N. V. Sastry. 1990. Preliminary immunotherapeutic trials in bovine mastitis. Livest. Adviser 15:3-10.

Kutila, T., L. Suojala, T. Lehtolainen, H. Saloniemi, L. Kaartinen, M. Tahti, K. Seppala, and S. Pyorala. 2004. The efficacy of bovine lactoferrin in the treatment of cows with experimentally induced Escherichia coli mastitis. J. Vet. Pharmacol. Ther. 27:197-202.

Leitner, G., Y. Pinchasov, E. Morag, Y. Spanier, S. Jacoby, D. Eliau, and J. Pitcovski. 2013. Immunotherapy of mastitis. Vet. Immunol. Immunopathol. 153:209-216.

Lohius, J. A. C. M., W. v. Leeuwen, J. H. M. Verheijden, A. Brand, and A. S. J. P. A. M. v. Miert. 1989. Effect of steroidal antiinflammatory drugs on Escherichia coli endotoxin-induced mastitis in the cow. J. Dairy Sci. 72:241-249.

Lohuis, J. A., T. van Werven, A. Brand, A. S. van Miert, E. Rohde, B. Ludwig, P. Heizmann, and W. F. Rehm. 1991. Pharmacodynamics and pharmacokinetics of carprofen, a non-steroidal antiinflammatory drug, in healthy cows and cows with Escherichia coli endotoxin-induced mastitis. J. Vet. Pharmacol. Ther. 14:219-229.

Masson, H. 2006. Enquête sur le traitement des mammites cliniques en agriculture biologique en Bretagne. Utilisation de l'aromathérapie. Vol. DVM thesis. École Nationale Vétérinaire de Nantes, Nantes, France.

Mathie, R. T., and J. Clausen. 2014. Veterinary homeopathy: Systematic review of medical conditions studied by randomised placebocontrolled trials. Vet. Rec. 175:373-381.

Mathie, R. T., and J. Clausen. 2015a. Veterinary homeopathy: Meta-analysis of randomised placebo-controlled trials. Homeopathy 104:3-8.

Mathie, R. T., and J. Clausen. 2015b. Veterinary homeopathy: Systematic review of medical conditions studied by randomised trials controlled by other than placebo. BMC Vet. Res. 11:236.

Mathie, R. T., M. V. Wassenhoven, J. Jacobs, M. Oberbaum, J. Frye, R. K. Manchanda, H. Roniger, F. Dantas, L. A. Legg, J. Clausen, S. Moss, J. R. Davidson, S. M. Lloyd, I. Ford, and P. Fisher. 2016. Model validity and risk of bias in randomised placebo-controlled trials of individualised homeopathic treatment. Complement. Ther. Med. 25:120-125.

Mayer, M., C. R. Vogl, M. Amorena, M. Hamburger, and M. Walkenhorst. 2014. Treatment of organic livestock with medicinal plants: A systematic review of European ethnoveterinary research. Forsch. Komplementmed. 21:375-386.

Mueller, K. 2004. Comparison of homoeopathic and antibiotic treatment of clinical mastitis. Pages 113-115 in Proc. British Mastitis Conference. Institute of Animal Health, Newbury, UK.

Pham, B., T. P. Klassen, M. L. Lawson, and D. Moher. 2005. Language of publication restrictions in systematic reviews gave different results depending on whether the intervention was conventional or complementary. J. Clin. Epidemiol. 58:769-776.

Pinchasov, Y., J. Spanier, G. Lietner, J. Pitcovsky, E. Morag, D. Raymond, and A. Gordon. 2005. The safety and efficacy of the immunotherapy treatment Y-complex in a clinical mastitis study under field conditions. Isr. J. Vet. Med. 60:82-85.

Pinedo, P., H. Karreman, H. Bothe, J. Velez, and C. Risco. 2013 Efficacy of a botanical preparation for the intramammary treatment of clinical mastitis on an organic dairy farm. Can. Vet. J. $54: 479-484$.

Roberson, J. R. 2012. Treatment of clinical mastitis. Vet. Clin. North Am. Food Anim. Pract. 28:271-288.
Roberson, J. R., L. D. Warnick, and G. Moore. 2004. Mild to moderate clinical mastitis: Efficacy of intramammary amoxicillin, frequent milk-out, a combined intramammary amoxicillin, and frequent milk-out treatment versus no treatment. J. Dairy Sci. 87:583-592.

Ruegg, P. L. 2009. Management of mastitis on organic and conventional dairy farms. J. Anim. Sci. 87(Suppl.):43-55.

Saini, V., J. T. McClure, D. Leger, S. Dufour, A. G. Sheldon, D. T. Scholl, and H. W. Barkema. 2012. Antimicrobial use on Canadian dairy farms. J. Dairy Sci. 95:1209-1221.

Santman-Berends, I. M., J. M. Swinkels, T. J. Lam, J. Keurentjes, and G. van Schaik. 2016. Evaluation of udder health parameters and risk factors for clinical mastitis in Dutch dairy herds in the context of a restricted antimicrobial usage policy. J. Dairy Sci. 99:2930-2939.

Sargeant, J. M., A. Rajic, S. Read, and A. Ohlsson. 2006. The process of systematic review and its application in agri-food public-health. Prev. Vet. Med. 75:141-151.

Sato, S., H. Hori, and K. Okada. 1999. Effect of vitamin B2 on somatic cell counts in milk of clinical Staphylococcus aureus mastitis. J. Vet. Med. Sci. 61:569-571.

Sears, P. M., and K. K. McCarthy. 2003. Diagnosis of mastitis for therapy decisions. Vet. Clin. North Am. Food Anim. Pract. 19:93-108.

Suojala, L., H. Simojoki, K. Mustonen, L. Kaartinen, and S. Pyorala. 2010. Efficacy of enrofloxacin in the treatment of naturally occurring acute clinical Escherichia coli mastitis. J. Dairy Sci. 93:19601969.

Van Eenennaam, A. L., I. A. Gardner, J. Holmes, L. Perani, R. J. Anderson, J. S. Cullor, and W. M. Guterbock. 1995. Financial analysis of alternative treatments for clinical mastitis associated with environmental pathogens. J. Dairy Sci. 78:2086-2095.

Vangroenweghe, F., L. Duchateau, P. Boutet, P. Lekeux, P. Rainard, M. J. Paape, and C. Burvenich. 2005. Effect of carprofen treatment following experimentally induced Escherichia coli mastitis in primiparous cows. J. Dairy Sci. 88:2361-2376.

Varshney, J. P., and R. Naresh. 2005. Comparative efficacy of homeopathic and allopathic systems of medicine in the management of clinical mastitis of Indian dairy cows. Homeopathy 94:81-85.

Wagner, S. A., and M. D. Apley. 2003. Pharmacodynamics of isoflupredone acetate in an endotoxin-induced mastitis model. J. Dairy Sci. 86:792-798.

Wagner, S. A., and M. D. Apley. 2004. Effects of two anti-inflammatory drugs on physiologic variables and milk production in cows with endotoxin-induced mastitis. Am. J. Vet. Res. 65:64-68.

Werner, C., A. Sobiraj, and A. Sundrum. 2010. Efficacy of homeopathic and antibiotic treatment strategies in cases of mild and moderate bovine clinical mastitis. J. Dairy Res. 77:460-467.

Williamson, J. H., and S. J. Lacy-Hulbert. 2014. Lack of efficacy of homeopathic therapy against post-calving clinical mastitis in dairy herds in the Waikato region of New Zealand. N. Z. Vet. J. 62:8-14.

Yeiser, E. E., K. E. Leslie, M. L. McGilliard, and C. S. Petersson-Wolfe. 2012. The effects of experimentally induced Escherichia coli mastitis and flunixin meglumine administration on activity measures, feed intake, and milk parameters. J. Dairy Sci. 95:4939-4949.

Zhen, Y. H., L. J. Jin, X. Y. Li, J. Guo, Z. Li, B. J. Zhang, R. Fang, and Y. P. Xu. 2009. Efficacy of specific egg yolk immunoglobulin (IgY) to bovine mastitis caused by Staphylococcus aureus. Vet. Microbiol. 133:317-322.

Zimov, J. L., N. A. Botheras, W. P. Weiss, and J. S. Hogan. 2011. Associations among behavioral and acute physiologic responses to lipopolysaccharide-induced clinical mastitis in lactating dairy cows. Am. J. Vet. Res. 72:620-627.

Ziv, G., and F. Longo. 1991. Comparative clinical efficacy of ketoprofen and flunixin in the treatment of induced $E$. coli endotoxin mastitis in lactating dairy cows. Pages 207-208 in Proc. Mammites des vaches laitières, Société française de Buiatrie. Polygone, Toulouse, France. 


\section{APPENDIX BOOLEAN SEARCH STRATEGIES USED IN A SYSTEMATIC REVIEW OF THE LITERATURE ON NON-ANTIMICROBIAL TREATMENTS OF CLINICAL MASTITIS IN DAIRY COWS}

The search strategy for each database was a combination of different components describing these 3 concepts of interest: disease (i.e., clinical mastitis), population (i.e., dairy cows), and intervention (i.e., non-antimicrobial treatment). Each component included either key words (all databases), thesaurus terms (CAB Abstracts), or medical sub-heading (MESH) terms (Medline) describing the targeted concepts. Components are described below. Moreover, only articles published after 1969 were retained.

\section{SEARCH STRATEGIES}

\section{Web of Science (https://login.webofknowledge.com)}

Search performed on June 3, 2014.

((clinical OR acute OR toxic) AND mastitis AND (cow OR cows OR cattle* OR bovine) AND (acupressure OR acupuncture or acutherapy OR alternative medicine* OR anti-inflammatory OR antiinflammatory OR aromatherap* OR ascorbic acid OR aspirin OR bach flower OR bacteriocin* OR bacteriophage OR biocell* OR biologic* OR biological treatment* OR botanical medicine* OR chinese medicine* OR chiroprati* OR clay OR complementary medicine* OR endobacteria OR energy therap* OR essential oil* OR fatty acid* OR flunixin OR frequent milk out OR frequent milking out OR herbal drug* OR herbal medicine* OR herbal remed* OR homeopath* OR hydrotherap* OR hyperimmune serum OR immune serum OR immune stimula* OR immunobooster OR immunostimula* OR immunotherap* OR immuno modulatory OR ketoprofen OR lactococcus lactis OR lantibiotic OR laser therap* OR medicinal plant* OR meloxicam OR natural products OR nisin or nonantibiotic* OR non antibiotic* OR non-steroidal anti-inflammatory agent* OR nsaid OR omega OR orbeseal OR organic* OR osteopath* OR oxytocin OR phage OR physical therap* OR physiotherap* OR phytotherap* OR plant extract* OR prebiotic* OR probiotic* OR teat sealer* OR therapeutic massage* OR traditional medicine* OR traditional chinese medicine* OR vitamin*))

\section{CAB Abstracts (http://ovidsp.ovid.com/autologin.cgi)}

Search performed on June 3, 2014.

((clinical OR acute OR toxic) AND ((mastitis) OR (mastitis/)) AND ((cow OR cows OR cattle*
OR bovine) $\underline{O R}$ (bovidae/ OR cattle/ OR cows/)) AND ((acupressure or acupuncture OR acutherapy OR alternative medicine* OR anti-inflammatory OR anti-inflammatory OR aromatherap* OR ascorbic acid OR aspirin OR bach flower OR bacteriocin* OR bacteriophage or biocell* OR biologic*biological treatment* OR botanical medicine*OR chinese medicine* OR chiroprati* OR clay OR complementary medicine* OR endobacteria OR energy therap* OR essential oil* OR fatty acid* OR flunixin OR frequent milk out OR frequent milking out OR herbal drug* OR herbal medicine* OR herbal remed* OR homeopath* OR hydrotherap* OR hyperimmune serum OR immune serum OR immune stimula* OR immunobooster OR immunostimula* OR immunotherap* OR immuno modulatory OR ketoprofen OR lactococcus lactis OR lantibiotic OR laser therap* OR medicinal plant* OR meloxicam OR natural products OR nisin OR nonantibiotic* OR non antibiotic* OR non-steroidal anti-inflammatory agent* OR nsaid OR omega OR orbeseal OR organic* OR osteopath* OR oxytocin OR phage OR physical therap* OR physiotherap* OR phytotherap* OR plant extract* OR prebiotic* OR probiotic* OR teat sealer* OR therapeutic massage* OR traditional medicine* ${ }^{*}$ R traditional chinese medicine* OR vitamin*) $O R$ (acupuncture.sh. OR alternative medicine.sh. OR antiinflammatory agents/ OR ascorbic acid/ OR aspirin/ OR bacteriocins/ OR bacteriophages/ OR biological treatment.sh. OR clay/ OR complementary medicine. sh. OR complementary and alternative medicine.sh. OR essential oils.sh. OR fatty acids.sh. OR flunixin/ OR herbal drugs.sh. OR homeopathy.sh. OR immune serum/ OR ketoprofen/ OR lactococcus lactis/ OR medicinal plants.sh. OR natural products.sh. OR nisin/ OR non-steroidal antiinflammatory agents.sh. OR oxytocin/ OR plant extracts.sh. OR prebiotics/ OR probiotics/ OR traditional medicine.sh. OR traditional medicine/ OR traditional chinese medicine)))

\section{Medline (https://www.ncbi.nlm.nih.gov/pubmed)}

Search performed on June 3, 2014.

((clinical mastitis OR acute mastitis OR toxic mastitis) AND ((mastitis) $\underline{O R}$ ("mastitis, bovine"[Mesh] OR "mastitis"[Mesh])) AND ((cow OR cows OR cattle* OR bovine) $\underline{O R}$ ("cattle"[Mesh] OR bovine"[Mesh])) AND ((acupressure OR acupuncture OR acutherapy OR alternative medicine* OR anti-inflammatory OR anti-inflammatory OR aromatherap* OR ascorbic acid OR aspirin OR bach flower OR bacteriocin* OR bacteriophage OR biocell* OR biological treatment* OR biological product* OR botanical medicine* OR chinese medicine* OR chiroprati* OR clay OR complementary medicine* OR endobacteria OR energy therap* OR es- 
sential oil* OR fatty acid* OR flunixin OR frequent milk out OR frequent milking out OR herbal drug* OR herbal medicine* OR herbal remed* OR homeopath* OR hydrotherap* OR hyperimmune serum OR immune serum OR immune stimula* OR immunobooster OR immunostimula* OR immunotherap* OR immuno modulatory OR ketoprofen OR lactococcus lactis OR lantibiotic OR laser therap* OR medicinal plant* OR meloxicam OR nisin OR nonantibiotic* OR non antibiotic* OR non-steroidal anti-inflammatory agent* OR natural product* OR nsaid OR omega OR orbeseal OR organic* OR osteopath* OR oxytocin OR phage OR physical therap* OR physiotherap* OR phytotherap* OR plant extract* OR prebiotic* OR probiotic* OR teat sealer* OR therapeutic massage* OR traditional medicine* OR traditional chinese medicine* OR vitamin*[all]) $\underline{O R}$ ("acupuncture"[MeSH Terms] OR "anti-Inflammatory agents, non-Steroidal"[Mesh] OR "aromatherapy"[Mesh] OR "ascorbic Acid"[Mesh]
OR "aspirin"[Mesh] OR "bacteriocins"[Mesh] OR "bacteriophages"[Mesh] OR "biological products"[Mesh] OR "chiropractic"[Mesh] OR "complementary therapies"[Mesh] OR "fatty acids"[Mesh] OR "fatty acids, omega-3"[Mesh] OR "fatty acids, omega6"[Mesh] OR "flunixin"[Supplementary Concept] OR "homeopathy"[MeSH Terms] OR "ISCOMs"[Mesh] OR "ketoprofen"[Mesh] OR "laser therapy"[Mesh] OR "laser therapy, low-level"[Mesh] OR "manipulation, chiropractic"[Mesh] OR "medicine, chinese traditional"[Mesh] OR "medicine, traditional"[Mesh] OR "meloxicam"[Supplementary Concept] OR "nisin"[Mesh] OR "nisin F, lactococcus lactis" [Supplementary Concept] OR "oils, volatile"[Mesh] OR "organic agriculture"[Mesh] OR "oxytocin"[Mesh] OR "physical therapy modalities"[Mesh] OR "phytotherapy"[MeSH Terms] OR "plant extracts"[Mesh] OR "plants, medicinal"[Mesh] OR "prebiotics"[Mesh] OR probiotics Mesh))) 